\title{
Retrospective study of imported falciparum malaria in French paediatric intensive care units
}

\author{
Justine Lanneaux, ${ }^{1}$ Stéphane Dauger, ${ }^{1}$ Luu-Ly Pham, ${ }^{2}$ Jérôme Naudin, ${ }^{1}$ Albert Faye, ${ }^{3}$ \\ Yves Gillet, ${ }^{4}$ Emmanuelle Bosdure, ${ }^{5}$ Ricardo Carbajal, ${ }^{6}$ François Dubos, \\ Renaud Vialet, ${ }^{8}$ Gérard Chéron, ${ }^{9}$ François Angoulvant ${ }^{9,10}$
}

- Additional material is published online only. To view please visit the journal online (http://dx.doi.org/10.1136/ archdischild-2015-309665).

For numbered affiliations see end of article.

\section{Correspondence to} Dr François Angoulvant, Service des Urgences Pédiatriques, Hôpital NeckerEnfants malades, 149 Rue de Sèvres, Paris 75015, France; francois.angoulvant@aphp.fr

Received 30 August 2015 Revised 4 May 2016 Accepted 10 May 2016 Published Online First 8 June 2016

\section{SLinked}

- http://dx.doi.org/10.1136/ archdischild-2016-310977

\section{CrossMark}

To cite: Lanneaux J,

Dauger S, Pham L-L, et al. Arch Dis Child

2016;101:1004-1009.

\section{ABSTRACT}

Objective The World Health Organization (WHO) severity criteria for paediatric Plasmodium falciparum (Pf) malaria are based on studies in countries of endemic malaria. The relevance of these criteria for other countries remains unclear. We assessed the relevance of these criteria in an industrialised country.

Design Retrospective case-control study.

Setting Eight French university hospitals, from 2006 to 2012.

Patients Children with Pf malaria admitted to paediatric intensive care units (cases: $n=55$ ) or paediatric emergency departments (controls: $n=110$ ).

Main outcome measures Descriptive analysis of WHO severity criteria and major interventions (mechanical ventilation, blood transfusion, fluid challenge, treatment of cerebral oedema, renal replacement therapy).

Thresholds were set by receiver operating characteristics curve analysis.

Results Altered consciousness (71\% vs $5 \%$ ), shock ( $24 \%$ vs $1 \%)$, renal failure ( $20 \%$ vs $1 \%)$, anaemia $<50 \mathrm{~g} / \mathrm{L}(7 \%$ vs $2 \%)$, acidosis (38\% vs $0 \%$ ), bilirubin level $>50 \mu \mathrm{mol} / \mathrm{L}(25 \%$ vs $8 \%$ ) and parasitaemia $>10 \%$ $(30 \%$ vs $8 \%)$ were more frequent in cases $(p<0.01)$. All these criteria were associated with major interventions $(p<0.001)$. Respiratory distress (six cases), and hypoglycaemia (two cases) were infrequent. Thrombocytopenia $<50000 / \mathrm{mm}^{3}$ (46\% vs $7 \%$ ) and anaemia (haemoglobin concentration $<70 \mathrm{~g} / \mathrm{L}(41 \%$ vs $13 \%))$ were more frequent in cases $(p<0.0001)$.

Conclusions The WHO severity criteria for paediatric Pf malaria are relevant for countries without endemic malaria. The infrequent but severe complications also provide a timely reminder of the morbidity and mortality associated with this condition worldwide. In non-endemic countries haemoglobin $<70 \mathrm{~g} / \mathrm{L}$ and platelet count $<50000 / \mathrm{mm}^{3}$ could be used as additional criteria to identify children needing high level of care.

\section{INTRODUCTION}

Plasmodium falciparum (Pf) malaria is a major international health problem. It can be severe and associated with high mortality. ${ }^{1}$ Despite a $25 \%$ decrease in mortality over the last decade, World Health Organization (WHO) has reported 216 million cases of malaria and 655000 deaths from malaria annually worldwide, mostly related to Pf; ${ }^{2}$ $91 \%$ of the deaths occurred in Africa and $86 \%$ of these deaths were in children under the age of 5 years. France is the industrialised country most affected by imported Pf malaria: in 2011, 4000

\section{What is already known on this topic?}

- Plasmodium falciparum is responsible for severe cases of imported malaria in children, some requiring intensive care in industrialised countries.

- The World Health Organization (WHO) severity criteria for paediatric Plasmodium falciparum malaria are based on studies performed in countries in which malaria is endemic.

\section{What this study adds?}

- The World Health Organization (WHO) severity criteria appear to be relevant to imported paediatric Plasmodium falciparum malaria: most children admitted to intensive care units had neurological complications.

- In non-endemic countries haemoglobin <70 g/L and platelet count $<50000 / \mathrm{mm}^{3}$ could be used as additional criteria to identify children needing high level of care.

cases were reported, $13.2 \%$ in children under the age of 15 years. $^{3} 4$

The severity of Pf malaria in children is assessed with the WHO severity criteria of 1990 , as revised in $2000 .^{5}$ These criteria are designed to alert clinicians to particularly severe cases and to guide treatment according to the level of care available. ${ }^{5}$ These criteria are essentially based on findings obtained in areas in which malaria is endemic (table 1$).^{5}$ The relevance of these criteria to adult cases in countries in which malaria is not endemic was recently assessed, ${ }^{4}$ but little is known about their relevance for children requiring malaria care in industrialised countries in which this disease is not endemic. ${ }^{36-8}$

The main objective of this retrospective study was to assess the relevance of WHO severity criteria for children hospitalised for Pf malaria in France, a country in which malaria is not endemic.

\section{METHODS}

Study design

Eight paediatric intensive care units (PICUs) and eight paediatric emergency departments from eight 
Table 1 WHO severity criteria for children ${ }^{3}$

\begin{tabular}{|c|c|c|}
\hline WHO severity criteria, 2000 & Frequency* & Prognosis* \\
\hline $\begin{array}{l}\text { Coma }(G C S<11) \text {-impaired consciousness } \\
(\mathrm{GCS}<15) / \text { prostration }\end{array}$ & +++ & $+++/+$ \\
\hline Seizure & +++ & + \\
\hline Respiratory distress & +++ & +++ \\
\hline Jaundice & +++ & ++ \\
\hline Circulatory collapse & + & +++ \\
\hline Abnormal bleeding & \pm & +++ \\
\hline Pulmonary oedema & \pm & +++ \\
\hline Haemoglobinuria & \pm & + \\
\hline Renal failure & + & ++ \\
\hline Hypoglycaemia (<2.2 mmol/L) & +++ & +++ \\
\hline Acidosist & +++ & +++ \\
\hline Severe anaemia $(\mathrm{Hb}<50 \mathrm{~g} / \mathrm{L})$ & +++ & + \\
\hline Hyperlactataemia (>5 mmol/L) & ++ & +++ \\
\hline Hyperparasitaemia ( $\geq 4 \%$ non-immune subject) & + & \pm \\
\hline
\end{tabular}

French university hospitals participated in this national retrospective case-control study. These hospitals admit most of the French paediatric Pf malaria cases. ${ }^{3}$ All children admitted to PICUs for imported Pf malaria between January 2006 and December 2012 were included as cases.

\section{Inclusion criteria and definitions}

The inclusion criteria for cases were: (1) PICU admission, (2) laboratory-confirmed malaria and (3) age under 18 years. Each case was matched with two controls: the two previous patients with paediatric Pf malaria admitted to the paediatric emergency department of the same hospital immediately before the case. Thus, only two criteria were used to match cases and controls: centre and disease. Pf malaria was defined as fever associated with the presence of asexual Pf forms in the blood. Severity was assessed with the 2000 revision of the WHO severity criteria. ${ }^{5}$ According to WHO guidelines (2012 and 2015), hyperparasitaemia $>10 \%$ and a bilirubin concentration $>50 \mu \mathrm{mol} / \mathrm{L}$ are severity criteria, and an age $<3$ years is associated with poor prognosis. ${ }^{19}$

WHO also defined haemoglobin concentration $<50 \mathrm{~g} / \mathrm{L}$ as a severity criterion. ${ }^{15}$ However, a threshold of $70 \mathrm{~g} / \mathrm{L}$ is recommended for blood transfusion in low-transmission settings. ${ }^{1} 9$ We tested both thresholds. Circulatory collapse was defined as a combination of hypotension (systolic blood pressure $<80 \mathrm{~mm} \mathrm{Hg}$ for children over 5 years of age and $<60 \mathrm{~mm} \mathrm{Hg}$ for children under 5 years of age) and poor perfusion (cool peripheries, prolonged capillary refill time: eg, $>3 \mathrm{~s}$, and a rapid, feeble pulse). ${ }^{5}$ We also assessed thrombocytopenia, which is known to be associated with severe anaemia. ${ }^{9}$ Severity was assessed by counting deaths and major interventions, defined as the transfusion of blood products, including red blood cells and platelets, fluid challenge, catecholamine infusion, mechanical ventilation, treatment of cerebral oedema with hypertonic mannitol or an intracranial monitoring device, and renal replacement therapy.

\section{Data collection}

Cases and controls were ascertained by the diagnostic codes in the medical and administrative database of the French hospital system (Programme de médicalisation des systèmes d'information), which uses the 10th International Classification of Diseases, ${ }^{10}$ and laboratory databases. For each patient, medical history (current and past conditions), sociodemographic data, clinical and laboratory findings were collected retrospectively from medical charts, on a standardised case report form. Disease progression and treatment were also recorded.

\section{Analysis}

We first compared cases and controls. We then compared groups of patients with and without major interventions. We assessed the relevance of WHO severity criteria by determining whether they were associated with at least one major intervention being performed. We also assessed the relevance of anaemia and thrombocytopenia thresholds by determining whether they were associated with at least one major intervention being performed and/or with any other WHO severity criteria (excluding anaemia and blood product transfusion). A descriptive analysis was performed. Continuous variables were described with medians and IQRs (medians (first; third quartile)), or means and SDs (mean \pm SD) for variables with a Gaussian distribution. $\chi^{2}$ test and Fisher's exact tests were used to analyse categorical variables and Wilcoxon rank sum tests were used for continuous data, as appropriate. We considered $\mathrm{p}$ values $<0.05$ to be statistically significant. If the observed rate for controls was 0 , we calculated an approximate OR by adding 0.5 to each cell. ${ }^{11}$ Thrombocytopenia was analysed dichotomously after setting a relevant threshold by screening sensitivityspecificity pairs by receiver operating characteristics (ROC) curve analysis. Missing data were not imputed. Statistical analyses were performed with Stata V.11.2 software (STATA, College Station, Texas, USA).

\section{RESULTS}

We included 165 children with imported Pf malaria: 55 cases and 110 controls.

\section{General characteristics}

General characteristics at admission are presented in table 2.

The proportion of children under 3 years of age did not differ significantly between groups (cases $25 \%$ vs controls $18 \%$, $\mathrm{p}=0.28)$. Most patients in both groups were born in France (cases $86 \%$ vs controls $88 \%$ ). All cases of Pf malaria were acquired in sub-Saharan Africa, including four cases from the islands of Comoros and Madagascar. Previous imported disease following overseas travel was recorded for 9 cases (malaria in 6 cases) and 16 controls (malaria in 14 cases). Appropriate antimalarial prophylaxis with good compliance was reported for very few patients in each group (table 2).

\section{WHO severity criteria for cases and controls}

WHO severity criteria by group (cases vs controls) are presented in table 3.

Twelve patients with neurological disorders had Glasgow coma scores $<11$, nine had seizures and three had cerebral oedema. The neurological disorder necessitated mechanical ventilation in seven patients. Brain imaging was performed for nine cases (CT scans in five and MRI in four) and two controls (one CT scan and one MRI). During hospitalisation, 19 cases suffered circulatory collapse and underwent fluid challenge; 6 required catecholamine infusion.

All cases of acute renal failure resolved quickly and none required renal replacement therapy. ${ }^{12}$ Other WHO severity 
Table 2 General characteristics of 165 children admitted with Plasmodium falciparum malaria

\begin{tabular}{|c|c|c|c|}
\hline Characteristics & Cases $(n=55)$ & Controls $(n=110)$ & p Value \\
\hline Boys, $n(\%)$ & $29 / 55(52.7 \%)$ & $65 / 110(59.1 \%)$ & $0.44^{*}$ \\
\hline Age (months), median (IQR) & $94(33-147)$ & $87(39-134)$ & 0.96 \\
\hline Infants ( $<3$ years old), $\mathrm{n}(\%)$ & $14 / 55(25.5 \%)$ & $20 / 110(18.2 \%)$ & $0.28^{*}$ \\
\hline Born in countries with endemic malaria, $n(\%)$ & $8 / 55(14.5 \%)$ & $13 / 110(11.8 \%)$ & $0.63^{*}$ \\
\hline Duration of overseas travel (days), median (IQR) (missing data $n=16$ ) & $46(29-90)$ & $51(31-62)$ & $0.96+$ \\
\hline Duration of symptoms before admission (days), median (IQR) (missing data $\mathrm{n}=27$ ) & $4(2-6)$ & $2(1-5)$ & $0.04 \dagger$ \\
\hline Antimalarial chemoprophylaxis, $\mathrm{n}(\%)$ & & & $0.40 \ddagger$ \\
\hline Appropriate & $3 / 52(5.8 \%)$ & $10 / 104(9.6 \%)$ & \\
\hline Inappropriate and/or low compliance & $22 / 52(42.3 \%)$ & $53 / 104(51.0 \%)$ & \\
\hline None & $27 / 52(51.9 \%)$ & $43 / 104(41.4 \%)$ & \\
\hline Duration of PICU stay (days), median (IQR) & $2(1-3)$ & - & \\
\hline Duration of hospital stay (days), median (IQR) & $6(4-7)$ & $1(0-2)$ & $<0.01 \dagger$ \\
\hline
\end{tabular}

criteria, such as respiratory distress, hypoglycaemia and coagulation disorders, were infrequent among both cases and controls (table 3). Haemoglobin concentration was rarely below $50 \mathrm{~g} / \mathrm{L}$, in either group (cases 9\% vs controls 1\%), but frequently below $70 \mathrm{~g} / \mathrm{L}$ (cases $41 \%$ vs controls $13 \%$ ).

The ROC curve of platelet levels in cases and controls had an inflexion point at $50000 / \mathrm{mm}^{3}$, with a sensitivity of $48 \%$ and a specificity of $91 \%$ (see online supplementary file 1). Thrombocytopenia, defined by a threshold of 50000 platelets/ $\mathrm{mm}^{3}$, was more frequent in cases than in controls $(47 \%$ vs $7 \%$; table 3).
Management and course of falciparum malaria

Numbers and types of major interventions in each group are reported in table 4 .

More cases than controls required at least one major intervention $(62 \%$ vs $10 \% ; \mathrm{p}<0.0001)$. Mechanical ventilation was required for a neurological indication in 7 of the 10 cases; there were 18 red blood cell transfusions and 6 platelet transfusions. Eleven controls underwent red blood cell transfusion, with additional fluid challenge in one patient.

No major intervention was performed in 21 cases on PICU (38\%), including 14 with impaired consciousness admitted for

Table 3 WHO severity criteria in 165 children with imported falciparum malaria

\begin{tabular}{|c|c|c|c|c|}
\hline Characteristics & Cases $(n=55)$ & Controls $(n=110)$ & OR $(95 \% \mathrm{Cl})^{*}$ & p Value* \\
\hline \multicolumn{5}{|l|}{ WHO characteristics } \\
\hline Impaired consciousness $(\mathrm{GCS}<15)$ & $39 / 55(70.9 \%)$ & $5 / 110(4.5 \%)$ & 51 (18 to 149$)$ & $<0.0001$ \\
\hline Seizure & 9/55 (16.4\%) & $0 / 110(0 \%)$ & $45(2.6$ to 792$) \dagger$ & $<0.0001$ \\
\hline Circulatory collapse & $13 / 55(23.6 \%)$ & $1 / 110(0.9 \%)$ & 34 (4.0 to 266$)$ & $<0.0001$ \\
\hline Respiratory distress & $4 / 55(7.3 \%)$ & $2 / 110(1.8 \%)$ & $4.2(0.8$ to 24$)$ & 0.08 \\
\hline Coagulation disorders & $2 / 55(3.5 \%)$ & $1 / 110(0.9 \%)$ & $4.1(0.4$ to 46$)$ & 0.21 \\
\hline Jaundice & $8 / 55(14.6 \%)$ & $14 / 110(12.7 \%)$ & $1.2(0.5$ to 3.0$)$ & 0.75 \\
\hline Renal failure & $11 / 55(20.0 \%)$ & $1 / 110(0.9 \%)$ & 27 (3.4 to 217) & $<0.0001$ \\
\hline Haemoglobinuria & $6 / 55(10.9 \%)$ & $3 / 110(2.7 \%)$ & 4.4 (1.0 to 18$)$ & 0.03 \\
\hline Hypoglycaemia $(<2.2 \mathrm{mmol} / \mathrm{L})$ & $2 / 49(4.1 \%)$ & $0 / 67(0 \%)$ & $7.1(0.3$ to 151$) \dagger$ & 0.10 \\
\hline Anaemia $(<50 \mathrm{~g} / \mathrm{L})$ & $4 / 54(7.4 \%)$ & $2 / 101(2.0 \%)$ & $4.0(0.7$ to 22$)$ & 0.01 \\
\hline Parasitaemia (> 10\%) & $15 / 50(30.0 \%)$ & $8 / 98(8.2 \%)$ & 4.8 (1.9 to 12$)$ & $<0.001$ \\
\hline Acidosisł & $18 / 47(38.3 \%)$ & $0 / 79(0 \%)$ & 100 (5.8 to 1708$) \dagger$ & $<0.0001$ \\
\hline Hyperlactataemia $>5 \mathrm{mmol} / \mathrm{L}$ & $5 / 29(17.2 \%)$ & $0 / 3(0 \%)$ & $1.6(0.1$ to 35$) \dagger$ & 0.43 \\
\hline Bilirubin concentration $>50 \mu \mathrm{mol} / \mathrm{L}$ & $10 / 40(25.0 \%)$ & $5 / 65(7.7 \%)$ & $4(1.3$ to 13$)$ & 0.01 \\
\hline \multicolumn{5}{|l|}{ Additional characteristics } \\
\hline Anaemia (<70 g/L) & $22 / 54(40.7 \%)$ & $13 / 101(12.9 \%)$ & $4.7(2.1$ to 10$)$ & $<0.0001$ \\
\hline Thrombocytopenia (<50 000 platelets $/ \mathrm{mm}^{3}$ ) & $25 / 54(46.3 \%)$ & $7 / 102(6.9 \%)$ & 11.7 (4.6 to 30$)$ & $<0.0001$ \\
\hline$C$ reactive protein $(\mathrm{mg} / \mathrm{L})$, mean $\pm S D$ & $126 \pm 78$ & $67 \pm 53$ & & $<0.0001 \S$ \\
\hline White cell counts $\left(\right.$ cells $\left./ \mathrm{mm}^{3}\right)$, mean \pm SD & $10.5 \pm 8.3$ & $7.6 \pm 3.1$ & & $0.06 \S$ \\
\hline Neutrophil counts (cells $/ \mathrm{mm}^{3}$ ) mean \pm SD & $5.7 \pm 3.9$ & $4.3 \pm 2.2$ & & $0.04 \S$ \\
\hline
\end{tabular}


Table 4 Major interventions in 165 children with imported falciparum malaria

\begin{tabular}{lccc}
\hline $\mathbf{n}(\%)$ & Cases $(\mathbf{n}=55)$ & Controls $(\mathbf{n}=\mathbf{1 1 0})$ & $\mathbf{p ~ V a l u e}$ * \\
\hline Mechanical ventilation & $10 / 55(18.2 \%)$ & $0 / 110(0 \%)$ & $<0.0001$ \\
Fluid challenge & $19 / 55(34.6 \%)$ & $1 / 110(0.9 \%)$ & $<0.0001$ \\
Catecholamine infusion & $6 / 55(10.9 \%)$ & $0 / 110(0 \%)$ & $<0.001$ \\
Blood product transfusion & $23 / 55(41.8 \%)$ & $11 / 110(10 \%)$ & $<0.0001$ \\
Treatment of cerebral oedema & $3 / 55(5.5 \%)$ & $0 / 110(0 \%)$ & 0.04 \\
Renal replacement therapy & $0 / 55(0 \%)$ & $0 / 110(0 \%)$ & - \\
Number of major interventions performed & & \\
0 & $21 / 55(38.2 \%)$ & $99 / 110(90.0 \%)$ & \\
1 & $20 / 55(36.4 \%)$ & $10 / 110(9.1 \%)$ & \\
2 & $7 / 55(12.7 \%)$ & $1 / 110(0.9 \%)$ & \\
At least 3 & $7 / 55(12.7 \%)$ & $0 / 110(0 \%)$ & \\
\hline
\end{tabular}

Cases: Children hospitalised in paediatric intensive care units (PICUs); Controls: Children not hospitalised in PICUs.

*Fisher's exact test.

neurological monitoring only. Four of the seven remaining cases were admitted to the PICU for intravenous quinine administration, as the oral route was not available. For the other three cases $(5 \%)$, we were unable to identify a major intervention or organ dysfunction justifying PICU admission.

No deaths occurred. Sequelae were observed in four cases: toe ischaemia, postencephalitic coma, pyramidal hypertonia and focal segmental glomerulosclerosis. Intravenous treatment was administered to 49 cases (89\%): 45 received intravenous quinine and 4 intravenous artesunate.

Concomitant bacterial infections were diagnosed in three $(5 \%)$ cases (meningitis in one patient and pneumonia in two) and seven $(6 \%)$ controls (pyelonephritis in one, pneumonia in two and skin infections in four). Empirical antibiotic treatment was prescribed in $23(42 \%)$ cases and 10 (9\%) controls.
Assessment of WHO severity criteria on the basis of the major intervention performed

At least one major intervention was performed in 45 (27\%) patients, including 34 cases. Assessments of WHO severity criteria in patients undergoing major intervention are reported in table 5. Impaired consciousness, haemodynamic disorders, renal failure, anaemia (haemoglobin $<70 \mathrm{~g} / \mathrm{L}$ ) and thrombocytopenia $\left(<50000\right.$ platelets $\left./ \mathrm{mm}^{3}\right)$ were frequent and strongly associated with major interventions.

We also compared patients with severe disease, defined as symptoms or signs from the WHO severity criteria (excluding anaemia) and patients undergoing any major intervention (excluding blood product transfusion). Fifty-seven of the 165 patients included fulfilled this definition (cases 46/55 (83.6\%) and controls $11 / 110(10 \%) ; \chi^{2}$ test $\left.\mathrm{p}<0.0001\right)$. Analysis of the association of haemoglobin, platelet and parasitaemia levels with major interventions (excluding blood product transfusion) and/or severity signs (excluding anaemia) showed that severe malaria was strongly associated with anaemia $<70 \mathrm{~g} / \mathrm{L}(18 / 54$ (33.3\%) vs $17 / 101(16.8 \%), \mathrm{p}=0.02)$, thrombocytopenia $<50$ 000 platelets $/ \mathrm{mm}^{3}(26 / 55$ (47.3\%) vs 6/101 (5.9\%), $\mathrm{p}<0.0001)$, parasitaemia $>10 \%(15 / 52(28.9 \%)$ vs $8 / 96(8.3 \%), \mathrm{p}<0.001)$ (see online supplementary table S1). Furthermore, blood product transfusion rates increased with decreasing haemoglobin concentration (mean: $65 \mathrm{~g} / \mathrm{L}(\mathrm{SD} \pm 0.40)$ vs 10.1 (SD \pm 0.18$)$, Wilcoxon rank sum test $\mathrm{p}<0.0001$ ) and platelet levels (mean: 92 platelets $/ \mathrm{mm}^{3}(\mathrm{SD} \pm 19)$ vs $159(\mathrm{SD} \pm 13)$, Wilcoxon rank sum test $\mathrm{p}<0.0001$ ) (see online supplementary table $\mathrm{S} 2$ ).

\section{DISCUSSION}

This multicentre study on paediatric imported Pf malaria describes the frequency with which the 2005 WHO severity criteria $^{5}$ and those of subsequent revisions ${ }^{19}$ were met, and their relevance. This is the largest study to date of paediatric cases admitted to PICUs for Pf malaria in an industrialised country in

Table 5 WHO severity criteria in patients with and without major interventions in165 children with imported falciparum malaria

\begin{tabular}{|c|c|c|c|c|}
\hline Characteristics, n (\%) & $\begin{array}{l}\geq 1 \text { Major } \\
\text { interventions }(n=45)\end{array}$ & $\begin{array}{l}0 \text { Major } \\
\text { intervention }(n=120)\end{array}$ & $\begin{array}{l}\text { OR } \\
(95 \% \mathrm{Cl})^{*}\end{array}$ & p Value* \\
\hline \multicolumn{5}{|l|}{ WHO characteristics } \\
\hline Impaired consciousness (GCS $<15)$ & $27 / 45(60.0 \%)$ & $17 / 120(14.2 \%)$ & 9.1 (3.9 to 21.5 ) & $<0.0001$ \\
\hline Seizure & $5 / 45(11.1 \%)$ & $4 / 120(3.4 \%)$ & $3.6(0.9$ to 14.2$)$ & 0.05 \\
\hline Circulatory collapse & $14 / 45(31.1 \%)$ & $0 / 120(0 \%)$ & 111 (6.4 to 1911)t & $<0.0001$ \\
\hline Respiratory distress & $4 / 45(8.9 \%)$ & $2 / 119(1.7 \%)$ & 5.7 (1.0 to 32.6$)$ & 0.27 \\
\hline Coagulation disorders & $2 / 45(4.4 \%)$ & $1 / 120(0.8 \%)$ & $5.5(0.5$ to 62.6$)$ & 0.12 \\
\hline Jaundice & $12 / 45(26.7 \%)$ & $10 / 120(8.3 \%)$ & $4.0(1.6$ to 10.1$)$ & $<0.01$ \\
\hline Renal failure & $10 / 45(22.2 \%)$ & $2 / 120(1.7 \%)$ & 16.9 (3.5 to 80.6$)$ & $<0.0001$ \\
\hline Haemoglobinuria & $5 / 45(11.1 \%)$ & $4 / 120(3.4 \%)$ & $3.6(0.9$ to 14.2$)$ & 0.05 \\
\hline Hypoglycaemia (<2.2 mmol/L) & $2 / 39(5.1 \%)$ & $0 / 77(0 \%)$ & $10(0.5$ to 221$) \dagger$ & 0.05 \\
\hline Anaemia (<50 g/L) & $6 / 45(13.3 \%)$ & $0 / 110(0 \%)$ & $36(2$ to 661$) \dagger$ & $<0.0001$ \\
\hline Parasitaemia (>10\%) & $14 / 43(32.6 \%)$ & $9 / 105(8.6 \%)$ & $5.1(2.0$ to 13.1$)$ & $<0.001$ \\
\hline Acidosis $\ddagger$ & $14 / 41(34.2 \%)$ & $4 / 84(4.8 \%)$ & 10.4 (3.1 to 34.2 ) & $<0.0001$ \\
\hline Hyperlactataemia (>5 mmol/L) & $4 / 23(17.4 \%)$ & $1 / 9(11.1 \%)$ & $1.7(0.2$ to 17.5$)$ & 0.66 \\
\hline Bilirubin concentration $>50 \mu \mathrm{mol} / \mathrm{L}$ & $11 / 34(32.4 \%)$ & $4 / 71(5.6 \%)$ & $8.0(2.3$ to 27.6$)$ & $<0.001$ \\
\hline \multicolumn{5}{|l|}{ Additional characteristics } \\
\hline Anaemia (<70 g/L) & $27 / 45(60.0 \%)$ & $8 / 110(7.3 \%)$ & 19.1 (7.5 to 48.7$)$ & $<0.0001$ \\
\hline Thrombocytopenia ( $<50000$ platelets $/ \mathrm{mm}^{3}$ & $22 / 45(48.9 \%)$ & $10 / 111(9.0 \%)$ & 9.7 (4.0 to 23.2 ) & $<0.0001$ \\
\hline
\end{tabular}

*Fisher's exact test.

$†$ An approximate $\mathrm{OR}$ was calculated by adding 0.5 to each cell.

$\ddagger$ Acidosis was defined as $\mathrm{HCO}_{3}{ }^{-}$concentration $<15 \mathrm{mmol} / \mathrm{L}$ and/or base excess $>-10$ and/or $\mathrm{pH}<7.35$

GCS, Glasgow Coma Scale. 
which malaria is non-endemic. This study covered a long time period (2006-2012) in France, where PICUs are readily accessible. We found that WHO severity criteria were generally relevant for imported Pf malaria in children. Disease severity, as assessed on the basis of the major interventions performed, was strongly correlated with the presence of WHO severity criteria, including impaired consciousness, circulatory collapse, renal failure, hyperparasitaemia and severe anaemia.

We identified two other studies of paediatric malaria cases admitted to PICUs in industrialised countries in which malaria is not endemic. ${ }^{13}{ }^{14}$ Garbash et al ${ }^{13}$ studied 29 children in the UK between 2004 and 2008, and Hay et al ${ }^{14}$ studied 15 children in France between 1990 and 1995. Thirty years separate the first patient included in these studies and the last patient included in our study, but the results were similar, with the frequency of severity criteria differing between countries with and without endemic malaria.

Impaired consciousness was the criterion most frequently observed in cases (71\%) and in children requiring at least one major intervention (60\%). It was more frequent than the 20-30\% generally reported for severe paediatric malaria in countries with endemic malaria, 2515 but its frequency was consistent with the two previous studies performed in European PICUs. ${ }^{13}{ }^{14}$ However, the threshold defining impaired consciousness applied may have been too low. Indeed, a large retrospective study of 19560 children with malaria reported neurological involvement in up to $48 \%$. $^{16}$

By contrast to WHO data, ${ }^{2}{ }^{5}$ but consistent with other published data, ${ }^{13}{ }^{17}$ circulatory collapse was also frequent in cases (24\%). Fluid challenge was performed in line with current French recommendations, despite recent controversy surrounding the use of this approach in African children. ${ }^{18}$

Renal failure, listed among WHO severity criteria and in the most recent consensus, pRIFLE (Pediatric Risk, Injury, Failure, Loss, End Stage Renal Disease), ${ }^{12}$ was identified in $20 \%$ of cases. It was transient and did not require renal replacement therapy, but was strongly correlated with major interventions. Indeed, acute renal failure seemed to be an independent prognostic factor. ${ }^{19}$

Severe anaemia (haemoglobin concentration $<50 \mathrm{~g} / \mathrm{L}$ ), according to WHO severity criteria, was rare, despite the large number of blood transfusions. Our patients probably underwent transfusion before the threshold of $50 \mathrm{~g} / \mathrm{L}$ was reached, as suggested by the haemoglobin levels of the patients receiving blood products in this study. ${ }^{20}$ During the study period there was no hospital policy to transfuse below a specific threshold. Transfusions were based on paediatric intensivist's decision. This discrepancy between endemic, ${ }^{2} 5$ and imported Pf cases has been reported before. ${ }^{8} 1321$ A threshold of $70 \mathrm{~g} / \mathrm{L}$ for severe anaemia seems to be more consistent with the routine blood transfusion practices of French PICUs, ${ }^{20}$ and with WHO recommendations for blood transfusion in low-transmission settings. ${ }^{1} 922$

Some of the WHO severity criteria (respiratory distress syndrome, oedema, coagulation disorders and hypoglycaemia) were less frequent in our population than in WHO reports. ${ }^{2} 5$ Respiratory distress syndrome due to acidosis is frequent in areas of endemic malaria, ${ }^{15}{ }^{17}$ and $23 \%$ of cases with both acidosis and impaired consciousness are fatal. ${ }^{19}$ These patients clearly have more severe disease than our cases. ${ }^{8} 13$

By contrast, some studies in countries with endemic malaria have reported hypoglycaemia in $10-20 \%$ of children with Pf malaria. ${ }^{17} 23$ However, hypoglycaemia may be aggravated by altered nutritional status in developing countries. ${ }^{24}$

Thrombocytopenia $\left(<50000\right.$ platelets $\left./ \mathrm{mm}^{3}\right)$ is not included in the WHO severity criteria but may be of value. It was strongly correlated with cases $(\mathrm{OR}=11.7$ (4.6-30)) and major interventions $(\mathrm{OR}=9.7$ (4.0-23.2)). Thrombocytopenia has repeatedly been linked to severity in studies in countries both with, ${ }^{15} 25$ and without endemic malaria. ${ }^{7}$

The therapeutic management of malaria changed with the authorisation of intravenous artesunate use by the French health product agency in $2011 .^{3}$ Intravenous artesunate is now recommended as the first line treatment for severe Pf malaria. ${ }^{26}$ Four patients received this treatment in 2012, and no adverse effects were detected during the weekly monitoring of haemoglobin levels for a month, following the description of haemolysis elsewhere. $^{26} 27$

\section{Limitations}

The retrospective nature of the study and the limited casecontrol matching create several limitations. At each centre, cases were matched with the two consecutive patients immediately preceding the case as controls. We did not match for age, which is itself recognised as a factor for malaria severity. ${ }^{5} \quad 9 \quad 15$ Unfortunately, this study was not powerful enough for in vestigations of the degree of dependence and correlation between age and other severity factors. Cases and controls were identified from laboratory and medical administrative databases, which probably identified all those with malaria. However, we cannot exclude the possibility of biases, with some of the less severe cases not being identified, resulting in a lack of complete case ascertainment or a systematic deviation of our result from the true value. Nevertheless, the largest French cities (Paris, Lyons, Marseilles, Lille) were covered and this study included more than half the cases of severe paediatric malaria occurring in mainland France from 2006 to 2012, and most of the cases admitted to PICUs. ${ }^{3}$ Some data, such as lactataemia, were missing for many patients. Children with less severe disease probably undergo fewer additional tests, but we did not impute the missing data. Assessments were carried out on admission to paediatric emergency department for controls and on admission to PICU for cases. The clinical status of cases at presentation should have influenced the decision to transfer the child to PICU and the need for major interventions. However, the time from initial admission to the paediatric emergency department to PICU admission did not exceed 12 hours for any of the cases.

In a prospective study in the UK and Ireland, only a quarter of the patients with severe imported paediatric Pf malaria required intensive care. ${ }^{7}$ This calls into question our use of major interventions for severity assessment. Blood transfusion may occur outside the PICU, as in $10 \%$ of the controls in our study. However, blood transfusion can have adverse effects in patients with malaria, ${ }^{28}$ and careful follow-up is required. ${ }^{9}$ Moreover, a haemoglobin concentration $<70 \mathrm{~g} / \mathrm{L}$ has already been used to define anaemia in assessments of severe malaria in another country in which malaria is not endemic. ${ }^{22}$

\section{CONCLUSION}

Our results confirm that the WHO severity criteria are generally relevant to imported malaria cases in industrialised countries in which malaria is not endemic, particularly as concerns the importance of neurological complications. The infrequent but severe complications also provide a timely reminder of the reasons for which this condition is such a major cause of morbidity and mortality worldwide. The identification of patients with severe Pf malaria is essential, to ensure that they receive appropriate care. ${ }^{5}$ In non-endemic countries haemoglobin $<70 \mathrm{~g} / \mathrm{L}$ and platelet count $<50000 / \mathrm{mm}^{3}$ could be used as additional criteria to identify children needing high level of care. 


\section{Author affiliations}

'Department of Paediatric Intensive care, AP-HP, Hôpital Robert Debré, Université Paris Diderot, Sorbonne Paris Cité, Paris, France

${ }^{2}$ Paediatric Emergency Department, AP-HP, Hôpital Bicêtre, Université Paris Sud, Le Kremlin-Bicêtre, France

${ }^{3}$ Department of General Paediatrics, AP-HP, Hôpital Robert Debré, Université Paris Diderot, Sorbonne Paris Cité, Paris, France

${ }^{4}$ Paediatric Emergency Department, HCL, HFME Lyon, Université de Lyon 1, Lyon, France

${ }^{5}$ Department of Paediatrics, APHM, CHU Timone Enfants, Marseille, France

${ }^{6}$ Paediatric Emergency Department, AP-HP, Hôpital Armand Trousseau, Université Pierre et Marie Curie Paris 6, Paris, France

${ }^{7}$ Paediatric Emergency and Infectious Diseases Departments, Université de Lille, Hôpital R. Salengro, Centre Hospitalier Régional Universitaire de Lille, Lille, France ${ }^{8}$ Paediatric Intensive Care Department, APHM, CHU Nord, Marseille, France ${ }^{9}$ Paediatric Emergency Department, AP-HP, Hôpital Necker-Enfants Malades, Université Paris Descartes, Sorbonne Paris Cité, Paris, France

${ }^{10}$ Clinical Epidemiology Unit ECEVE, INSERM UMR 1123, AP-HP, Hôpital Robert Debré, Université Paris Diderot, Sorbonne Paris Cité, Paris, France

Acknowledgements The authors thank all the physicians and technicians of the laboratories responsible for performing the Pf tests, the French Paediatric Intensive and Emergency Care Society (GFRUP), the French National Reference Centre for Imported and Autochthonous Malaria, and the following physicians for their contributions, which made this work possible: Serge Gottot, Laurence Morin, Pierre Mornand.

Contributors Study concept and design: JL, SD, FA; data acquisition: JL, L-LP, JN, $A F, Y G, E B, G C, R C, F D, R V$; data analysis and interpretation: JL, SD, L-LP, JN, $A F$, $Y G, E B, G C, R C, F D, R V, F A$; drafting of the manuscript: JL, SD, $F$; r revision of the manuscript: JL, SD, L-LP, JN, AF, YG, EB, GC, RC, FD, RV, FA; statistical analysis: JL, SD, FD, GC, FA; FA had full access to all the data in the study and takes responsibility for the integrity of the data and the accuracy of the data analysis.

Competing interests None declared.

Ethics approval Comité de Protection des Personnes, lle de France XI.

Provenance and peer review Not commissioned; externally peer reviewed.

Data sharing statement Data are available on request from francois. angoulvant@aphp.fr.

\section{REFERENCES}

1 World Health Organisation. Guidelines for the treatment of malaria. 3rd edn. Geneva: World Health Organization, Switzerland, 2015.

2 World Health Organisation. World Malaria Report 2011. Geneva: World Health Organization, Switzerland, 2011.

3 French National Reference Center for Imported and Autochthonous Malaria. Activity report 2011, France: Institut de Veille Sanitaire, Paris, 2012. Accessed at http://www. astrium.com/file/rapport_activites_cnr_paludisme_2011.pdf (accessed 2 june 2016).

4 Naudin J, Blondé R, Alberti C, et al. Aetiology and epidemiology of fever in children presenting to the emergency department of a French paediatric tertiary care centre after international travel. Arch Dis Child 2012;97:107-11.

5 World Health Organisation. Severe falciparum malaria. Trans R Soc Trop Med Hyg 2000;94(Suppl 1):S1-90.

6 Driessen GJ, Pereira RR, Brabin BJ, et al. Imported malaria in children: a national surveillance in the Netherlands and a review of European studies. Eur J Public Health 2008;18:184-8.
7 Ladhani S, Garbash M, Whitty CJ, et al. Prospective, national clinical and epidemiologic study on imported childhood malaria in the United Kingdom and the Republic of Ireland. Pediatr Infect Dis / 2010;29:434-8.

8 Ladhani S, Aibara RJ, Riordan FA, et al. Imported malaria in children: a review of clinical studies. Lancet Infect Dis 2007;7:349-57.

9 World Health Organisation. Management of severe malaria: a practical handbook. 3rd edn. Geneva: World Health Organization, Switzerland, 2012.

10 World Health Organization. International Statistical Classification of Diseases and Related Health Problems 10th Revision Version for 2010. Secondary International Statistical Classification of Diseases and Related Health Problems 10th Revision Version for 2010. 2010. http://www.who.int/classifications/

11 Glas AS, Lijmer JG, Prins MH, et al. The diagnostic odds ratio: a single indicator of test performance. J Clin Epidemiol 2003;56:1129-35.

12 Plötz FB, Bouma $A B$, van Wijk JA, et al. Pediatric acute kidney injury in the ICU: an independent evaluation of pRIFLE criteria. Intensive Care Med 2008;34:1713-17.

13 Garbash M, Round J, Whitty CJ, et al. Intensive care admissions for children with imported malaria in the United Kingdom. Pediatr Infect Dis I 2010;29:1140-2.

14 Hay F, Treluyer JM, Orbach $\mathrm{D}$, et al. [Severe malaria in children in intensive care. National survey 1990-1995]. Arch Pediatr 2000;7:1163-70.

15 Cserti-Gazdewich CM, Dhabangi A, Musoke C, et al. Inter-relationships of cardinal features and outcomes of symptomatic pediatric Plasmodium falciparum MALARIA in 1,933 children in Kampala, Uganda. Am J Trop Med Hyg 2013;88:747-56.

16 Idro R, Ndiritu M, Ogutu B, et al. Burden, features, and outcome of neurological involvement in acute falciparum malaria in Kenyan children. JAMA 2007:297:2232-40.

17 Dondorp AM, Fanello $\mathrm{Cl}$, Hendriksen IC, et al. Artesunate versus quinine in the treatment of severe falciparum malaria in African children (AQUAMAT): an open-label, randomised trial. Lancet 2010;376:1647-57.

18 Maitland K, Kiguli S, Opoka RO, et al. Mortality after fluid bolus in African children with severe infection. N Engl J Med 2011;364:2483-95.

19 von Seidlein L, Olaosebikan R, Hendriksen IC, et al. Predicting the clinical outcome of severe falciparum malaria in african children: findings from a large randomized trial. Clin Infect Dis 2012;54:1080-90.

20 Du Pont-Thibodeau G, Tucci M, Ducruet T, et al. Survey on stated transfusion practices in PICUs*. Pediatr Crit Care Med 2014;15:409-16.

21 Evans $A B$, Kulik $D$, Banerji $A$, et al. Imported pediatric malaria at the hospital for sick children, Toronto, Canada: a 16 year review. BMC Pediatr 2014;14:251.

22 Kiang KM, Bryant PA, Shingadia D, et al. The treatment of imported malaria in children: an update. Arch Dis Child Educ Pract Ed 2013;98:7-15.

23 Onyiriuka AN, Peter 00, Onyiriuka LC, et al. Point-of-admission hypoglycaemia among under-five Nigerian children with plasmodium falciparum malaria: prevalence and risk factors. Med I Islam Repub Iran 2012;26:78-84.

24 Sambany E, Pussard E, Rajaonarivo C, et al. Childhood dysglycemia: prevalence and outcome in a referral hospital. PLOS ONE 2013:8:e65193.

25 Gérardin P, Rogier C, Ka AS, et al. Prognostic value of thrombocytopenia in African children with falciparum malaria. Am J Trop Med Hyg 2002;66:686-91.

26 Zoller T, Junghanss $T$, Kapaun $A$, et al. Intravenous artesunate for severe malaria in travelers, Europe. Emerging Infect Dis 2011;17:771-7.

27 Kreeftmeijer-Vegter AR, van Genderen PJ, Visser LG, et al. Treatment outcome of intravenous artesunate in patients with severe malaria in the Netherlands and Belgium. Malar J 2012;11:102

28 Meremikwu M, Smith HJ. Blood transfusion for treating malarial anaemia. Cochrane Database Syst Rev 2000;(2):CD001475. 